\title{
THE BRUHAT ORDER OF THE SYMMETRIC GROUP IS LEXICOGRAPHICALLY SHELLABLE
}

\author{
PAUL H. EDELMAN
}

\begin{abstract}
The title theorem is proven. It then follows from a theorem of Björner that the simplicial complex of chains of this Bruhat order is shellable and thus Cohen-Macaulay. It is further established that this complex is a double cone over a triangulation of a sphere.
\end{abstract}

In this note we present an elementary proof that the Bruhat order of the symmetric group $S_{n}$ is lexicographically shellable and hence Cohen-Macaulay. Using a theorem of Verma we obtain as a corollary that $\Delta\left(S_{n}\right)$, the simplicial complex of chains of $S_{n}$, is a double cone over a triangulation of a sphere of dimension $\left(\begin{array}{l}n \\ 2\end{array}\right)-2$. We will employ the notation and terminology of Björner [2].

A finite poset $P$ is said to be bounded if it has a maximum and a minimum element, denoted $\hat{\mathbf{1}}$ and $\hat{0}$ respectively. It is called pure if all of its maximal chains are the same length and it is graded if it is both bounded and pure. The rank of $P$ is the length of a maximal chain. An element $x$ of a graded poset $P$ has a well-defined rank $\rho(x)$ equal to the length of an unrefinable chain from 0 to $x$ in $P$. If $P$ is bounded let $\bar{P}$ be the poset $P-\{\hat{0}, \hat{1}\}$.

The order complex $\Delta(P)$ of a poset $P$ is the simplicial complex of all chains in $P$. A poset is said to be shellable if $\Delta(P)$ is shellable. For the definition of a shellable complex see [2] or [4]. Similarly $P$ is called Cohen-Macaulay if $\Delta(P)$ is. See [1], [2] or [6] for the definition and significance of a Cohen-Macaulay complex.

Let $C(P)$ be the set of covering relations

$$
C(P)=\{(x, y) \in P \times P \mid x \text { is covered by } y\} .
$$

An edge-labeling of $P$ is a map $\lambda: C(P) \rightarrow \Lambda$ where $\Lambda$ is some poset. An edge-labeling corresponds to an assignment of elements of $\Lambda$ to the edges in the Hasse diagram of $P$. An unrefinable chain $x_{0}<x_{1}<\cdots<x_{n}$ in a poset with an edge-labeling $\lambda$ will be called increasing if $\lambda\left(x_{0}, x_{1}\right)<\lambda\left(x_{1}, x_{2}\right)<\cdots<$ $\lambda\left(x_{n-1}, x_{n}\right)$ in $\Lambda$.

With every saturated chain $c$, say with elements $x_{0}<x_{1}<\cdots<x_{n}$ of a poset $P$ having an edge-labeling $\lambda$, we associate the $n$-tuple

$$
\pi(c)=\left(\lambda\left(x_{0}, x_{1}\right), \lambda\left(x_{1}, x_{2}\right), \ldots, \lambda\left(x_{n-1}, x_{n}\right)\right) .
$$

We call $\pi(c)$ the Jordan-Hölder (J-H) sequence of $c$. Totally order $\Lambda^{n}$ by the lexicographic order: $\left(a_{1}, a_{2}, \ldots, a_{n}\right)$ precedes $\left(b_{1}, \ldots, b_{n}\right)$ if and only if $a_{i}<b_{i}$ in the first coordinate where they differ.

Received by the editors May 30, 1980.

1980 Mathematics Subject Classification. Primary 06A10.

(c) 1981 American Mathematical Society 0002-9939/81/0000-0308/\$02.00 
Let $\lambda$ be the edge-labeling of a graded poset $P$. $\lambda$ is said to be an L-labeling if it satisfies the following two conditions:

(i) In every interval $[x, y]$ of $P$ there is a unique increasing unrefinable chain $c$, $x=x_{0}<x_{1}<\cdots<x_{n}=y$.

(ii) The J-H sequence of the unique chain from (i) is lexicographically first among the J-H sequences of all unrefinable chains $x=z_{0}<z_{1}<\cdots<z_{n}=y$ in $[x, y]$. $P$.

A graded poset is called lexicographically shellable if there exists an $L$-labeling of

THEOREM (BJÖRNER [2]). If $P$ is lexicographically shellable then $P$ is shellable and hence Cohen-Macaulay.

We now define the Bruhat order on the symmetric group $S_{n}$. For our purposes $S_{n}$ will be the set of all permutations of the set $[n]=\{1,2, \ldots, n\}$. We will write $\pi \in S_{n}$ as a word $a_{1} a_{2} \ldots a_{n}$ in the letters $1,2, \ldots, n$. A reduction of $\pi$ is a permutation obtained from $\pi$ by interchanging some $a_{i}$ with some $a_{j}$ provided $i<j$ and $a_{i}>a_{j}$. Define $\sigma<\pi$ if $\sigma$ can be obtained from $\pi$ by a sequence of reductions. Figure 1 is a drawing of the poset $S_{3}$.

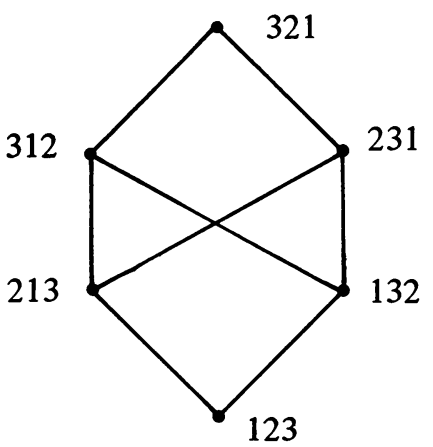

FIGURE 1

It is well known that the rank of a permutation $\pi$ in $S_{n}$ is the number of inversions in $\pi$, i.e. the number of pairs $(i, j)$ where $i<j$ and $a_{i}>a_{j}$. Thus if $\sigma$ is covered by $\pi$ then $\pi$ has one more inversion than $\sigma$. The rank of $S_{n}$ is $\left(\begin{array}{l}n \\ 2\end{array}\right)$.

THEOREM. $S_{n}$ is lexicographically shellable.

Proof. Let $Z$ be the set of ordered pairs $(i, j) \in[n] \times[n]$ such that $i<j$. Totally order $Z$ by $(i, j)<(r, s)$ if $i<r$ or if $i=r$ and $j<s$. Let $\lambda: C\left(S_{n}\right) \rightarrow Z$ be the labeling

$$
\lambda\left(\sigma_{1}, \sigma_{2}\right)=(i, j)
$$

if $i$ and $j$ are interchanged in $\sigma_{1}$ to obtain $\sigma_{2}$ and $i<j$. For example in $S_{3}$ we have $\lambda(123,213)=(1,2)$ and $\lambda(213,312)=(2,3)$.

We proceed to show that $\lambda$ is an $L$-labeling by first showing that in any interval $[x, y]$ the lexicographically first chain increases and then showing that there is a unique increasing chain in $[x, y]$. 
We will show that the lexicographically first chain increases by contradiction. There are a number of cases to consider. We will present one and leave the others to the reader. Let $c$ be the lexicographically first chain in $[x, y], x=\pi_{0}<\pi_{1}$ $<\cdots<\pi_{n}=y$ and suppose it has a decrease. Then there are three permutations $\pi_{r-1}<\pi_{r}<\pi_{r+1}$ such that

$$
\lambda\left(\pi_{r-1}, \pi_{r}\right)>\lambda\left(\pi_{r}, \pi_{r+1}\right) .
$$

Suppose $\lambda\left(\pi_{r-1}, \pi_{r}\right)=(i, j)$ and $\lambda\left(\pi_{r}, \pi_{r+1}\right)=(i, k)$ where $j>k$. Then the permutation $\pi_{r-1}$ looks like

$$
a_{1} a_{2} \ldots i \ldots j \ldots k \ldots a_{n}
$$

since the interchange $(i, j)$ must produce a cover. Define $\pi_{r}^{\prime}$ by interchanging $i$ and $k$ in $\pi_{r-1}$. $\pi_{r}^{\prime}$ covers $\pi_{r-1}$ and is covered by $\pi_{r+1}$. Moreover $\lambda\left(\pi_{r-1}, \pi_{r}^{\prime}\right)=(i, k)$. Since $(i, k)<(i, j)$ the chain $c^{\prime}$ with $\pi_{r}^{\prime}$ replacing $\pi_{r}$ in $c$ is lexicographically earlier than $c$. This is a contradiction.

There are other cases to consider when the labels $\lambda\left(\pi_{r-1}, \pi_{r}\right)$ and $\lambda\left(\pi_{r}, \pi_{r+1}\right)$ are disjoint. They are similar to the above argument.

What is left to show is that the increasing chain in the interval $[x, y]$ is unique. This follows from a series of remarks. Let $x=a_{1} a_{2} \ldots a_{n}$ and $y=b_{1} b_{2} \ldots b_{n}$. Define $p(r)=j$ if and only if $r=a_{j}$ and define $s(r)=j$ if and only if $r=b_{j}$. Let $i$ be the smallest number such that $p(i) \neq s(i)$.

REMARK 1. No number less than $i$ will appear in any label in $[x, y]$. Suppose this were not true. Then there is some label $(j, k)$ in $[x, y]$ where $j<i$ and $j$ is the smallest number appearing in such a label. Since $p(j)=s(j)$ when $j$ is interchanged with $k, j$ is moved to the right of the correct position for it in $y$. Since no smaller number appears as a label $j$ cannot be moved back to the left. Hence $j$ cannot appear in a label in $[x, y]$.

REMARK 2. $p(i)<s(i)$. This follows from an argument similar to that used in Remark 1.

REMARK 3. In an increasing chain, the first label contains $i$. The element $i$ must be switched sometime to get from $x$ to $y$ and since it is the smallest number it must occur first.

REMARK 4. If $\lambda\left(x, \pi_{1}\right)=(i, k)$ where $\pi_{1}$ is the second permutation in an increasing chain, then $p(k)<s(i)$. This follows from the same arguments as those used in Remark 1.

Let $j$ be the smallest number such that $i<j$ and $p(i)<p(j)<s(i)$.

REMARK 5. The first label on an increasing chain is $(i, j)$. Suppose this were not the case. By Remark 3 the first label involves $i$. Suppose it is $(i, k), k \neq j$. By Remark 4,p(k) $\leqslant s(i)$. If $p(j)<p(k)$ then the switch $(i, k)$ increases the number of inversions in the permutation by at least two, since $k>j>i$. So $(i, k)$ does not produce a cover. Hence $p(k)<p(j)$. But sometime $i$ and $j$ must switch, since $p(i)<p(k)<p(j)$. Then the label $(i, j)$ appears which forces a decrease. So the first label must be $(i, j)$.

Hence an increasing chain is uniquely determined. Since the lexicographically first chain increases, $\lambda$ is an $L$-labeling and the proof is complete. 
A graded poset is called Eulerian if in every interval $[x, y]$ the identity

$$
\sum_{x<z<y}(-1)^{\rho(z)-\rho(x)}=0
$$

holds. If $\boldsymbol{P}$ is Eulerian then $\overline{\boldsymbol{P}}$ also satisfies (*) for all intervals $[x, y]$. It is easily seen that every interval of rank 2 in an Eulerian poset is isomorphic to the poset in Figure 2. R. Stanley observed that if $P$ satisfies (*) and is of rank $k$ then $\Delta(P)$ is a pseudomanifold of dimension $k$, i.e. every $k-1$ face is contained in exactly two $k$ faces. From this observation we deduce

COROLlARY. $\Delta\left(\overline{S_{n}}\right)$ is a triangulation of a sphere of dimension $\left(\begin{array}{l}n \\ 2\end{array}\right)-2$.

Proof. Verma [7] has shown that $S_{n}$ is Eulerian. Hence $\Delta\left(\bar{S}_{n}\right)$ is a pseudomanifold. Since $\Delta\left(S_{n}\right)$ is shellable by the previous theorem so is $\Delta\left(\bar{S}_{n}\right)$. Since it is known that a shellable pseudomanifold is a sphere (see for example [3, p. 444]) the corollary is proven.

R. Proctor has extended the Theorem to the Bruhat orders of the other classical Weyl groups as well as their quotients by parabolic subgroups [5].

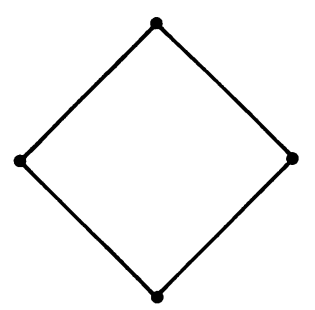

\section{FiguRE 2}

NOTE ADDED IN PROOF. Björner and Wachs have recently generalized the Theorem for all Coxeter groups modulo a parabolic subgroup (Bruhat orders of Coxeter groups and shellability, Report 1980-No. 20, Department of Mathematics, University of Stockholm).

\section{REFERENCES}

1. K. Baclawski, Cohen-Macaulay ordered sets, J. Algebra 63 (1980), 226-258.

2. A. Björner, Shellable and Cohen-Macaulay partially ordered sets, Trans. Amer. Math. Soc. 260 (1980), 159-183.

3. G. Danaraj and V. Klee, Shellings of spheres and polytopes, Duke Math. J. 41 (1974), 443-451.

4. Which spheres are shellable?, Algorithmic Aspects of Combinatorics, Ann. Discrete Math. 2 (1978), 33-52.

5. R. Proctor, Classical Bruhat orders are lexicographically shellable (in preparation).

6. R. Stanley, Cohen-Macaulay complexes, Higher Combinatorics, edited by M. Aigner, Reidel, Dordrecht and Boston, Mass., 1977, pp. 51-62.

7. D.-N. Verma, Möbius inversion for the Bruhat ordering on a Weyl group, Ann. Sci. Ecole Norm. Sup. (4) 4 (1971), 393-399.

Department of Mathematics, Massachusetts Institute of Technology, Cambridge, MasSACHUSETTS 02139

Current address: Department of Mathematics E1, University of Pennsylvania, Philadelphia, Pennsylvania 19104 